\title{
Fine motor deficits in reading disability and language impairment: same or different?
}

Several studies have found evidence of motor deficits in poor readers. There is no obvious reason for motor and literacy skills to go together, and it has been suggested that both deficits could be indicative of an underlying problem with cerebellar function and/or procedural learning. However, the picture is complicated by the fact that reading problems often co-occur with oral language impairments, which have also been linked with motor deficits. This raises the question of whether motor deficits characterise poor readers when language impairment has been accounted for - and vice versa. We considered these questions by assessing motor deficits associated with reading disability (RD) and language impairment (LI). A large community sample provided a subset of 9- to 10-year-olds, selected to oversample children with reading and/or language difficulties, to give 37 children with comorbid LI+RD, 67 children with RD only, 32 children with LI only, and 117 typicallydeveloping (TD) children with neither type of difficulty. These children were given four motor tasks that taxed speed, sequence, and imitation abilities to differing extents. Different patterns of results were found for the four motor tasks. There was no effect of RD or LI on two speeded fingertip tapping tasks, one of which involved sequencing of movements. LI, but not $\mathrm{RD}$, was associated with problems in imitating hand positions and slowed performance on a speeded peg-moving task that required a precision grip. Fine motor deficits in poor readers may be more a function of language impairment than literacy problems. 
Annie Brookman, Department of Experimental Psychology, University of Oxford, Oxford, UK Sarah McDonald, Department of Experimental Psychology, University of Oxford, Oxford, UK David McDonald, Department of Experimental Psychology, University of Oxford, Oxford, UK Dorothy V M Bishop, Department of Experimental Psychology, University of Oxford, Oxford, UK

Corresponding author:

Prof Dorothy V M Bishop,

Department of Experimental Psychology,

University of Oxford,

9, South Parks Road,

Oxford,

OX1 3UD,

UK.

01865271369

dorothy.bishop@psy.ox.ac.uk 


\section{Introduction}

2 It has been noted for many years that children who are poor readers may also show signs of

3 clumsiness and poor fine motor control. In an early epidemiological study, Rutter and Yule (1970)

4 found an excess of motor impairments in children who were poor readers relative to their IQ

5 ('specific reading retardation'), regardless of whether this was assessed by parental report, clinical

6 observation or direct assessment. This kind of observation has been used as evidence that reading

7 disability (RD) is not just the result of poor teaching, but has a neurological basis (Ramus, 2004).

8 However, the link between motor impairment and literacy problems remains poorly understood.

9 One complication is that it remains unclear whether motor impairments are a genuine

10 correlate of $\mathrm{RD}$, or whether they are linked more closely to other problems that co-occur with

11 poor reading. Many children diagnosed with RD (or 'developmental dyslexia') also have oral

12 language problems, but these may be overlooked if language is not formally assessed (Bishop \&

13 Snowling, 2004). Studies of children with language impairments (LI) provide ample evidence

14 that motor deficits are common in this population. These observations raise two related questions.

15 Will we find evidence of motor impairment if we focus only on poor readers who do not have

16 oral language problems? And if motor deficits are seen in children with combined reading and

17 language impairments, are they the same as those in children who read well despite oral language

18 problems? Because many children have both reading and language difficulties, the existing

19 literatures on RD and LI cannot answer these questions: we need a study of children who have

20 been explicitly assessed for both oral and written language abilities.

Another issue concerns how motor skills are measured. Previous studies have included

22 both fine and gross motor skills, tasks that stress speed versus those stressing precision, and tasks

23 that involve learning versus those that do not. We need to clarify whether RD and LI are

24 associated with distinct types of motor difficulty. The answer to this question has implications for 
25 our understanding of possible neurological underpinnings of children's language and literacy

26 problems.

Where motor deficits have been associated with RD or LI, two types of explanation have been proposed. It could be that the motor deficit co-occurs with other disorders because the causal factors that lead to RD and/or LI are correlated with causal factors that lead to motor problems. Typically this is interpreted at the neurobiological level; for instance, there could be a nonspecific factor, such as delay in myelination, that affects multiple systems at once, or there might be a more specific link, with a deficit affecting a brain region that is involved in both motor co-ordination and language learning, such as the cerebellum. Or the link may go beyond common etiology to involve shared underlying cognitive processes - for instance, language difficulties have been linked to limitations in speed of processing, in sequencing and in imitative capacity features that are implicated to different extent in different motor tasks. Our focus here is on fine motor skills that might be expected to relate to language impairment, insofar as they share these cognitive characteristics. For instance, theories of language impairment that implicate reduced speed of processing predict there will be links between reduced motor speed and slowed performance on language or literacy tasks that involve rapid processing. Thus, by pinpointing the nature of motor deficits that co-occur with reading or language difficulties, we may cast light on cognitive underpinnings of these disorders, clarifying whether they have similar origins.

We will first review what is known about different fine motor abilities in relation to reading and language impairments and then present new data on a large sample of children assessed for both language and literacy skills. We use the term 'reading disability' (RD) rather than 'dyslexia' to refer to children with specific problems in reading development, and 'language

47 impairment' (LI) to encompass those whose language development is significantly below age 
A number of speeded motor tasks have produced contradictory evidence in individuals

51 with reading difficulties. In some cases, poor readers are reported as slower than peers on tasks

52 such as peg-moving (Fawcett \& Nicolson, 1995; Francks et al., 2003; Stoodley \& Stein, 2006),

53 bead-threading (Fawcett \& Nicolson, 1995; Ramus, Pidgeon, \& Frith, 2003), foot-tapping

54 (Fawcett \& Nicolson, 1999) and finger-tapping (Morris et al., 1998). Fawcett and Nicolson

55

(1999) interpreted these findings as consistent with their theory of cerebellar impairment in RD,

as cerebellar patients show similar deficits in these tasks. However, other work has shown that

children with RD perform no differently to their peers on speeded tasks including peg-moving

(Irannejad \& Savage, 2012; Wimmer, Mayringer, \& Landerl, 1998), bead-threading (Irannejad \&

Savage, 2012; Savage \& Frederickson, 2006; White et al., 2006) foot-tapping (Gaysina,

Maughan, \& Richards, 2010) and speeded writing (Savage \& Frederickson, 2006). In addition,

Ramus et al. (2003) attributed the slowed bead-threading in their study to comorbid Attention

Deficit Hyperactivity Disorder (ADHD) or Developmental Co-ordination Disorder (DCD).

speeded motor tasks. An early demonstration of this was by Bishop and Edmundson (1987), who suggested that motor speed might be a marker of neurodevelopmental maturity. They found that on a peg-moving task many 4-year-olds with LIs improved from the impaired to the normal range over an 18-month follow-up period, with a close parallel between improvement in language skills and motor speed. They suggested a possible maturational lag in language impaired children, where the duration of the lag is related to the severity of LI. Bishop (2002) replicated the finding of slower peg-moving in an older group of LI children, and also demonstrated deficits on a

71 simple task that involved tapping a tally counter with the thumb as quickly as possible. Hill 72 (2001) suggested that slow motor performance might be part of a more general slowing of 
73 cognitive processing, which has been proposed to affect LI across several modalities (Kail,

74 1994).

75 Sequencing

76 Advocates of the cerebellar theory of RD have noted impairments of sequencing in

77 individuals with RD (Nicolson \& Fawcett, 2007). Consistent with this, Stoodley, Harrison and

78 Stein (2006) found that implicit motor learning was poor in adults with RD: on a serial reaction

79 time task, their speed did not improve when the sequence of stimuli was repeated, whereas

80 controls showed implicit learning. In a similar vein, an underlying deficit in the learning of

81 serial-order information has been described in RD, on the basis of impaired Hebbian learning

82 (Szmalec et al., 2011). The Hebb tasks involved implicit learning of the sequence of perceived

83 stimuli, rather than motor sequencing. However, if this kind of learning was impaired in LI or

$84 \mathrm{RD}$, it could lead to problems in automatizing the sequence of movements involved in motor

85 tasks. The finger to thumb task, which involves a repetitive sequence of hand movements, was

86 performed more slowly by children with RD in one study (Ramus et al., 2003). However, as with

87 the bead threading task, the authors suggest this may be due to comorbidity with other

88 developmental disorders. A further study found that children with RD performed as well as peers

89 on the finger to thumb task (White et al., 2006).

90 The ability to perform a sequence of actions has also been studied in children with LI.

91 Bishop and Edmundson (1987) noted that children with LI made more sequence errors in peg-

92 moving than controls; picking up pegs in the wrong order, or placing them in the wrong hole.

93 Hill, Bishop, and Nimmo-Smith (1998) interpreted greater errors in representational gesture

94 production as an inability to implement the precise sequence of movements in children with LI.

95 More recently, several studies have demonstrated impairments of implicit motor learning on the 
114 movements than typically developing (TD) children, showing a marked impairment even for

115 simple movements, whereas control children performed at ceiling levels. Consistent with this was

117 movements by much younger language-delayed children aged from 2 to 3 years. 
119 sequences, children with DCD or LI performed as well as peers, though interpretation of this 120 result was complicated by ceiling effects. On other tasks, Hill (1998) found difficulties in

121 production of representational gestures even when no imitation is required. When producing

122 representational gestures of familiar motor acts, children with LI and children with DCD made

123 more errors than age-matched children, and performed at a similar level to TD children who were

1244 years younger; however, this was found regardless of whether the child had to imitate the 125 gesture, or generate it from verbal command. This suggests that difficulties on imitation tasks

126 may be compounded by problems in conceiving and executing motor commands to produce 127 specific manual configurations. Hill (1998) concluded that when performing familiar actions, 128 kinaesthetic information may be especially important, and she suggested that the difficulties of 129 children with LI and those with DCD may have kinaesthetic origins.

Current Study

Our first question is whether motor deficits are associated with RD in children who do not have additional LI. We compared children with RD and those with LI to TD children on motor tests that varied in the demands they placed on speed, sequential ordering and imitation. No other

134 study has looked closely at the motor abilities of these two groups on the same tasks. Previous 135 research leads us to hypothesise that, regardless of whether they have additional RD, children 136 with LI will be impaired on tests of speeded motor movements (Bishop, 2002), pegmoving 137 (Bishop \& Edmundson, 1987), and motor imitation (Vukovic et al, 2010). Our hypothesis is that 138 previous associations with RD on some of these tasks may be due to inclusion of children with 139 LI, and that deficits should therefore not be seen in children with RD.

140 Second, we ask what kinds of motor skills are most closely linked with reading and/or 141 language abilities in the sample as a whole. We examined correlations between quantitative 
142 measures of speech, language and reading skills. This is an exploratory analysis that takes

143 advantage of the fact that we have a wide range of language, literacy and motor measures on a

144 sample of twins, and so can identify correlations that replicate in subsamples that take each

145 member of the twin pair separately. The aim of this analysis was to throw further light on the

146 nature of shared mechanisms between motor skills and language/literacy skills.

\section{Method}

148 Data collection conformed to the Declaration of Helsinki, and ethics approval was obtained from

149 Oxford University's Experimental Psychology Research Ethics Committee. Parents of

150 participating children gave informed consent, and children gave verbal assent, as agreed by the

151 Ethics Committee. Children were seen in a quiet room at home or school by a trained research

152 assistant. Motor tasks were interleaved within a battery of language and reading ability tests, in a 153 session lasting no longer than 2 hours.

154 Participants

155

The initial sample included 458 same-sex twins aged 9 to 10 years, recruited through the

156 Twins Early Development Study (TEDS), a non-clinical sample drawn from the general

157 population of twins born in England and Wales (Trouton, Spinath, \& Plomin, 1994). The

158 selection and categorisation of this particular subsample has been described in detail by Bishop,

159 McDonald, Bird, and Hayiou-Thomas (2009). All children were from White, English-speaking

160 families. As previously described, we oversampled children who had been identified as having

161 difficulties in language or literacy on previous waves of testing, so the numbers of impaired

162 children in this sample was higher than would be found in the general population. We excluded

163 from the sample those with low nonverbal ability (Block Design $<80, \mathrm{~N}=30$ ) or high nonverbal

164 ability (all 31 children with Block Design greater than 120 were excluded, plus 47 children with 
165 Block Design greater than 114 who would have been in the TD group: This was done to reduce 166 differences between groups in nonverbal ability). In addition, children were excluded on the 167 basis of failing a hearing screen $(\mathrm{N}=22)$, medical conditions $(\mathrm{N}=2)$, evidence of autistic 168 spectrum disorder $(\mathrm{N}=6)$, social deprivation $(\mathrm{N}=31$ children from a subsample studied by 169 Trzesniewski, Moffitt, Caspi, Taylor, \& Maughan, 2006), being cotwin of a child with major 170 exclusionary condition $(\mathrm{N}=13)$, or failure to complete the test battery $(\mathrm{N}=23)$. This left 253

171 participants who were aged 9 or 10 years at the time of testing (age $M=9.57 \mathrm{yr}, S D=.38$ ). The criteria used to categorise children were selected to be similar to those adopted by 173 Catts et al. (2005). Children were first grouped according to reading ability. Children were 174 classified as having RD if their average score on two subtests from the Test of Word Reading 175 Efficiency (TOWRE; Torgesen, Wagner, \& Rashotte, 1999) was below the 13th percentile.

176 Simulations of normal random data showed that assuming a correlation between the two subtests 177 of around .75, this cutoff will select around 11-12\% of the population. Children were also 178 categorised according to language ability, either as language typical or language impaired (LI).

179 Where a child had at least two scores more than $1.33 S D$ below the normative mean on five core 180 language measures (see below for details), they were categorised as LI. Assuming a correlation 181 between the language measures of around .5, this would select around $11 \%$ of the population.

182 Mean scores on the tests used to categorise children, and numbers in each group, are shown in 183 Table 1. An index of socio-economic status was available for $91 \%$ of the twin pairs, using 185 information gathered when families were first recruited to the Twins Early Development Study 186 (Petrill, Pike, Price, \& Plomin, 2004). This was the sum of z-scores derived from parental 187 educational and occupational status and age of mother at birth of eldest child, and had a mean of 
1880.10 and standard deviation of 0.72 in the whole TEDS sample. Missing values on this variable

189 were imputed with the sample mean.

\section{Language and reading tasks}

191 Core diagnostic tests. The battery of five core language tests, used to define LI, included 192 expressive and receptive tests of vocabulary and sentence processing: (1) Vocabulary was

193 measured using the Vocabulary subtest from the Wechsler Abbreviated Scale of Intelligence

194 (WASI: Wechsler, 1999); (2) The Understanding Directions subtest from the Woodcock-Johnson

195 III (Woodcock, McGrew, \& Mather, 2001) measured ability to carry out complex instructions; (3)

196 The Comprehension subtest from Expressive, Receptive and Recall of Narrative Instrument

197 (ERRNI; Bishop, 2004) measured ability to understand questions about a narrative; (4) Mean

198 Length of Utterance from the ERRNI was used as a measure of expressive syntactic complexity;

199 (5) Sentence Repetition from the NEPSY (Korkman, Kirk, \& Kemp, 1998) was used to assess

200 ability to repeat meaningful sentences of increasing length. Reading was assessed using the

201 TOWRE Phonological Decoding Efficiency and Word Reading Efficiency subtests (Torgesen et

202 al., 1999). These assess speeded reading of real words and nonwords. Scores on the two reading

203 subtests are highly correlated, and were averaged.

204 Supplementary language and literacy tests. Two additional subtests from the NEPSY, Oromotor

205 Skills and Nonword Repetition were used to assess speech production and phonological memory

206 respectively (Korkman et al., 1998). Rapid naming was assessed using an average score from the

207 Pictures and Digits Rapid Serial Naming subtests of the Phonological Assessment Battery

208 (Frederickson, Frith, \& Reason, 1997). Scores for Reading Accuracy, Comprehension and Rate

209 were obtained from a shortened version of the Neale Analysis of Reading Ability (Neale, 1997),

210 which assesses reading of meaningful texts. 
211 Nonverbal ability. The Block Design subtest from the WASI was administered as a measure of

212 nonverbal ability (Wechsler, 1999).

213 All tests are standardized, but scores were restandardized to a mean 100 and standard deviation of

21415 relative to a normative set of twins who were representative of the whole population, to ensure

215 comparability of norms across tests (see Bishop et al., 2009 for further details and for information

216 on reliability of measures).

\section{Motor tasks}

NEPSY Repetitive Fingertip Tapping (Korkman et al., 1998) was included as a simple measure of motor speed, which places few demands on sequencing or imitation. Children were required to tap their index finger to their thumb on the same hand, making a circular shape. The experimenter demonstrated, and children were instructed to repeat this action as fast as possible.

222 The time was noted for 32 correct taps. This procedure was administered using the child's preferred hand, and then repeated with the non-preferred hand. The mean time for 32 taps was inverted to give taps per second, so that proficient performance corresponded to a high score. sequential movement, but places few demands on imitation and does not require such fine dexterity as a peg-moving task. Children sequentially tapped their thumb to each finger of the same hand, from index to little finger. Participants were asked to repeat this sequence as fast as possible, and timed for 8 correct sequences. They first completed the sequences with their

230 preferred hand, and then their non-preferred hand. The mean time for eight sequences was

231 inverted to give sequences per second, so that proficient performance corresponded to a high 232 score. 
234 dexterity under time pressure. It was administered according to the procedure described by Tiffin

235 (1968). Children were given 30 seconds to move as many small pegs from a well into individual

236 peg holes (in a top-to-bottom line) as possible. This task was selected to assess precision grip,

237 which is known to depend on cerebellar activity (Monzée, Drew, \& Smith, 2004). Participants

238 completed the task twice with their preferred hand, then their non-preferred hand, giving a total

239 of 4 trials. The measure is the total number of pegs placed in holes.

NEPSY Imitating Hand Positions (Korkman et al., 1998) assesses the ability to imitate

241 hand and finger positions. Although there is a time limit on the test, the emphasis is on accuracy

242 rather than speed. Children were instructed to copy hand positions administered by the

243 experimenter. A maximum of 20 seconds was allowed for each of the 12 hand positions. One

244 point was awarded for each correct hand position within the time limit. Again children first

245 completed the task with their preferred hand, and then with their non-preferred hand.

246 Analytic approach

Previous research has not found reliable effects of language or literacy on difference in

skill of the two hands (Bishop, 1990, 2001), and so scores for preferred and non-preferred hands

were combined to form a composite score for each motor task. Scores were inspected and

transformations applied if necessary to correct for non-normality. A natural log transform was Hand Positions.

Our primary goal was to consider how language and reading status affected motor

254 performance on the different tasks, and so we included the binary categories of RD and LI as

255 fixed effects in SPSS multilevel linear models for each motor task. The interaction between LI 
256

257

258

259

260

261

262

263

264

265

266

267

268

269

270

271

272

273

274

275

276

277 literature (Smits-Engelsman \& Hill, 2012), some motor measures were significantly correlated 278

and RD was also tested to see whether the combination of both conditions had a greater impact than would be predicted from their separate effects. Sex was included as a covariate in the model to ensure that group differences were not attributable to this potential confounder, given that some previous studies have found sex differences in RD (Rutter et al., 2004) and a trend for more males was seen in the RD+LI group (see Table 1). Multilevel modelling allows one to conduct analyses that are analogous to conventional analysis of variance, but has greater flexibility. In particular, because our participants were twins, the individual observations were not independent. This was taken into account by including family membership as a random effect in the multilevel models (Kenny, Kashy, \& Cook, 2006). Effect sizes for main effects are reported as Cohen's d, based on difference in estimated marginal means divided by the pooled standard deviation. The SPSS script for the analysis is provided in Supplementary Table 1, together with more detailed explanation.

Analysis of RD and LI effects allows us to relate results to the prior literature, but these categories involve arbitrary subdivisions of continuous scales of language and reading ability. To explore the data in a more quantitative fashion, two-tailed Pearson correlations were computed for language and reading task standard scores with transformed motor scores, for supplementary as well as core diagnostic tests. Because of the large number of correlations computed, there is a risk of finding spurious associations, but the twin design of our study allowed for a natural replication study. Twins from each family were assigned randomly into twin group 1 or twin group 2 and correlations were run separately for each twin group. A correlation was regarded as replicable if it was statistically significant in both twin subsamples. Consistent with previous with nonverbal ability, and so the effect of nonverbal ability was partialled out to ensure than any 
279 significant associations were specific to language, and not attributable to general developmental 280 level.

\section{Results}

282 Means for each subgroup on the selection variables, nonverbal ability and SES are shown in 283 Table 1.

\section{Multilevel modelling}

Figure 1 shows mean raw scores on the four motor tests in relation to language and reading impairment. Log- or rank-transformed scores, as described above, were used in the analysis where appropriate to improve normality. F-ratios for the fixed effects and interaction are shown in Table 2. Repetitive Fingertip Tapping and Sequential Finger Tapping tasks, there was no significant effect of LI or RD, and no interaction between these factors. In contrast, on the Purdue Pegboard and NEPSY Imitating Hand Positions test there was a significant effect of LI. The effect of RD was not significant and there was no interaction between the two conditions.

\section{Correlations}

Figure 2 shows the correlations between cognitive tests and motor tests after partialling out Block Design. Results for the two subsamples of twins (each containing one member of a

297 twin pair, selected at random) are shown separately. The full sample was used for this analysis.

298 For a sample of this size, a correlation of .17 is significant at .05 level, a correlation of .23 is

299 significant at .01 level, and a correlation of .29 is significant at .001 level. None of the 300 correlations with finger-tapping were consistently found in both samples at the .05 level. 
302 correlations with speeded reading (TOWRE average) and the NARA subtests, as well as with

303 Sentence Repetition. For this task, the highest correlation in both subsamples was with NEPSY

304 Oromotor Sequences, suggesting that there may be a common core involvement of motor systems

305 in sequencing speech and finger movements.

306 The Purdue Pegboard task was reliably correlated with with Rapid Serial Naming, but 307 correlations with individual language tasks were mostly inconsistent from twin to twin. NEPSY

308 Imitating Hand Positions also showed an inconsistent pattern of correlations in the two

309 subsamples of twins. Only WASI Vocabulary was consistently significantly correlated with this 310 test in both subsamples.

\section{Discussion}

Our first question was whether motor deficits are associated with RD in children who do

313 not have additional LI. A large sample of twin children was divided into those with RD, those

314 with LI, those with RD+LI and those with no language or literacy problems (TD). When these

315 four groups were compared on performance on four motor tests, we found that LI status rather

316 than RD status was associated with poor performance on two measures. This suggested that

317 associations between motor impairments and RD may be largely driven by comorbid language

318 difficulties. Furthermore, motor tasks show different patterns of association with LI. This leads to

319 our second question: whether some specific aspects of motor function are linked with language

320 difficulties. We will consider the results in terms of the extent to which motor tasks stressed

321 speed, sequencing and imitation.

322 Speed 
324 Sequential Fingertip Tapping and the Purdue Pegboard. The simplest of these tasks, Repetitive

325 Fingertip Tapping, did not discriminate groups: children with RD or LI were as fast as TD

326 children on this measure. This contrasts with a previous study by Bishop (2002), who found

327 reduced speed on a thumb-tapping task in LI children. However, that task involved repeatedly

328 depressing the switch on a tally counter, a novel movement which some children found difficult

329 to do with one hand. Our current data show that if the task demands are reduced to the bare

330 minimum, children with developmental disorders of language and reading can perform as fast as

331 other children.

When the child had to sustain a repetitive sequence of finger movements, there was no

333 main effect of RD or LI in the categorical analysis. However, a correlational analysis on the

334 whole sample revealed reliable associations with the TOWRE measure of speeded reading, and

335 also with the three indices from the Neale Analysis of Reading Ability. This test also showed

336 significant associations with Sentence Repetition and Oromotor Sequences. These correlations

337 were all modest in size, and overall, children with RD did not do more poorly on sequential

338 finger movements than TD children of comparable nonverbal ability and social background.

The Purdue Pegboard, which involved quickly picking up and placing small metal components with a precision grip showed deficits in children with LI. This finding is compatible

341 with previous research that has found that peg-moving performance is impaired in children with

342 LI (Bishop \& Edmundson, 1987). Nevertheless, the effect size was small, and no overall

343 association between pegmoving and core language skills was found when the entire range of

344 ability was considered, and nonverbal ability was controlled for. 
347 intricate movements as fast as possible. Overall, the results do not support an account of

348 generally slowed processing in RD or LI. Rather, it seems that for children with LI, adding time

349 pressure to a task may reveal underlying difficulties with fine motor movements.

350 Sequencing

351

Problems in sequencing motor movements have been observed in children with LI doing

352 peg-moving (Bishop \& Edmundson, 1987) and gesture production (Hill et al., 1998), and

353 impaired sequence learning has been observed in serial reaction time tasks in both RD (Stoodley

354 et al., 2006) and LI (e.g. Tomblin et al., 2007). In the current study, the one task that involved

355 explicitly producing a sequence of motor movements, NEPSY Sequential Fingertip Tapping, did

356 not show a deficit in either RD or LI. Note, however, that the NEPSY Sequential Fingertip

357 Tapping task is very simple, and the sequence of movements is predictable. Furthermore, the

358 correlational analysis revealed that this motor task was associated with a measure of Oromotor

359 Sequences (repeatedly saying tongue-twisters). This task had not been included in the diagnostic

360 battery for LI, because it stresses articulation rather than language ability. This result suggests

361 that there may be overlap in neural systems involved in programming finger movements and

362 programming articulatory gestures, as has been previously suggested (Bishop, 2002). This

363 suggests it may be important to distinguish between the physical act of producing speech and

364 cognitive aspects of language function when looking for links with motor skill.

\section{Imitation}

Imitation tasks have shown that LI children successfully imitate fewer movements than peers (Vukovic et al., 2010), though for one study this was only true for familiar gestures (Hill,

368 1998). The current study confirmed that language impaired children correctly imitated fewer

369 hand positions, despite the fact that most of these were novel gestures. We are aware of no 
previous research on imitation abilities of children with $\mathrm{RD}$, which was not associated with

371 impaired imitation in the current study.

The interesting question raised by the imitation task is whether there is some supramodal

373 imitation ability that affects children's ability to learn language as well as their ability to imitate

374 gestures. Imitation involves perceiving a signal produced by another person and then translating

375 that observed percept into a motor programme for producing the same movement. Without

376 imitation ability, language could not be learned. Insofar as imitation has been an explicit focus of

377 research attention, this has mainly concerned children with autism, rather than LI. Deficits in

378 imitation are a hallmark of autism, and, in young autistic children, are predictive of receptive

379 language outcome (Charman et al. 2003). Our results suggest that milder imitative difficulties

380 may underlie slow learning in some children with LI.

Some neurological data support the link between language and imitation. Repetitive transcranial magnetic stimulation (rTMS) to Broca's area, well known for its role in speech production, interfered with imitation of action (Heiser et al., 2003). The stimulation did not significantly impair production of the same action when the cue to perform was spatial. This specific deficit in action imitation during rTMS suggests that certain parts of Broca's area have a role in action imitation. MRI has shown functional and structural abnormality in children with LI. Badcock et al. (2012) found reduced activation in Broca's area in children with LI during an inner speech task, and increased grey matter in this area compared to unaffected siblings and controls.

389 We can therefore speculate that the link between motor imitation deficits and LI reflects

390 developmental abnormality of Broca's area. This would fit with fMRI data showing that action

391 observation caused activation in Broca's area (Fadiga et al., 2006). Heiser et al. (2003) described

392 Broca's area as an area of shared neural mechanisms for communication; through language, 393 action imitation, and action recognition. 
Nevertheless, we need to be cautious in interpreting our results. When we considered

395

396

397

398

399

400

401

402

403

404

405

406

407

408

409

410

411 lower performance. For instance, the finger sequencing task was scored according to the speed

412 with which children completed 8 sequences. This measure alone cannot tell us whether some

413 children obtained lower scores because they made sequence errors, or because they were simply

414 slower but accurate. Similarly, deficits on peg-moving might involve dexterity or sequencing as

415 well as speed. Time pressure did not appear to be a major factor affecting performance in the test

416 of imitating hand positions, but nevertheless there was a time limit for each trial, and in future

417 studies it would be worth noting whether some children continued to attempt the posture after the 
418 limit expired. In future work it would be useful to devise tasks which are designed to separate the

419 requirements for imitation, sequence and speed, and also to focus on motor tasks that are known

420 to depend on specific motor systems. For instance, it would be of interest to identify tasks that

421 involve cortico-striatal versus cortico-cerebellar systems, and to look more directly at motor

422 learning as well as performance.

423 A second point is that such associations as exist between motor difficulties and

424 language/literacy problems are small in magnitude, especially when potential confounders have

425 been accounted for. The largest correlations between motor and language/literacy measures in

426 this sample were below .4, and the significant effect sizes seen in Table 2 were around .3. Such

427 effects are not easy to detect, especially in small samples, and may vary from sample to sample,

428 as is evident from the correlational analysis.

A third conclusion from this study is that RD and LI often co-occur, and motor impairments that are seen in poor readers may be more a function of their LI than their literacy

431 problems per se. We did not examine other comorbidities, such as attentional problems that often co-occur with both reading and language impairments, but there is some evidence that these too

433 can be a factor affecting whether or not motor impairments are observed (Raberger \& Wimmer,

434 2003; Ramus et al., 2003). It would be premature to conclude there are no motor impairments in

435 RD, given that our test battery was of necessity limited. Measures of balance, posture and muscle 436 tone were not included in our study, and their involvement in RD has been debated (e.g. Fawcett 437 \& Nicolson, 1999; Irannejad \& Savage, 2012; Needle, Fawcett, \& Nicolson, 2006; Rochelle \& 438 Talcott, 2006). However, the distinctive patterns of associated motor impairment obtained here 439 suggest we will obtain more coherent results if we assess both oral language and literacy skills 440 when looking for neurobiological bases of these developmental disorders. Where RD occurs in 
441 the absence of other comorbidities, motor difficulties are unlikely to be found on tests that stress

442 speed and dexterity of hand function.

Although we did not find convincing evidence of links between RD and motor skill, once

444 language had been taken into account, we did find significant associations with LI and

445 performance on a pegmoving task that stressed fine motor dexterity, and on imitation of hand

446 postures. These effects were not large, and were unlikely to be of practical importance for most

447 children. Nevertheless, such comorbidities are especially intriguging when they involve skills that

448 do not, on the surface, appear to have much in common. They may indicate common causes for

449 motor and language difficulties, which could give clues to etiology. For instance, the association

450 between problems with nonspeech oral movements and language difficulties in people with a

451 mutation of the FOXP2 gene has pointed to a role of this gene in the development of cortico-

452 basal ganglia circuits, which, in turn, has generated a rich body of research using animal models

453 (Enard, 2011). The etiology of common language and literacy problems is seldom as

454 straightforward as this, but by studying comorbid difficulties, we may uncover underlying

455 pathways that are implicated in speech, language and motor skill.

\section{Acknowledgements}

457 We thank the twins and their families and teachers who participated in this research. This study

458 would not have been possible without generous assistance of Robert Plomin, Bonamy Oliver,

459 Alexandra Trouton, and other staff from the Twins Early Development Study.

460 References 
461

462

463

464

465

466

467

468

469

470

471

472

473

474

475

476

477

478

479

480

481

482

483

484

485

486

487

488

489

490

491

492

493

494

495

496

497

498

499

500

501

502

503

504

505

506
Badcock, N. A., Bishop, D. V. M., Hardiman, M. J., Barry, J. G., \& Watkins, K. E. (2012). Colocalisation of abnormal brain structure and function in specific language impairment. Brain \& Language, 120(3), 310-320.

Bishop, D. V. M. (1990). Handedness, clumsiness and developmental language disorders. Neuropsychologia, 28(7), 681-690.

Bishop, D. V. M. (2001). Individual differences in handedness and specific speech and language impairment: Evidence against a genetic link. Behavior Genetics, 31(4), 339-351.

Bishop, D. V. M. (2002). Motor immaturity and specific speech and language impairment: Evidence for a common genetic basis. American Journal of Medical Genetics (Neuropsychiatric Genetics), 114(1), 56-63.

Bishop, D. V. M. (2004). Expression, Reception and Recall of Narrative Instrument (ERRNI). London: Psychological Corporation.

Bishop, D. V. M., \& Edmundson, A. (1987). Specific language impairment as a maturational lag: Evidence from longitudinal data on language and motor development. Developmental Medicine and Child Neurology, 29(4), 442-459.

Bishop, D. V. M., McDonald, D., Bird, S., \& Hayiou-Thomas, M. (2009). Children who read words accurately despite language impairment: Who are they and how do they do it? Child Development, 80(2), 593-605.

Bishop, D. V. M., \& Snowling, M. J. (2004). Developmental dyslexia and specific language impairment: same or different? Psychological Bulletin, 130(6), 858-86. doi:10.1037/00332909.130.6.858

Catts, H. W., Adlof, S. M., Hogan, T., \& Weismer, S. E. (2005). Are specific language impairment and dyslexia distinct disorders? Journal of Speech, Language, and Hearing Research, 48(6), 1378-1396.

Charman T, Baron-Cohen S, Swettenham J, Baird G, Drew A, and Cox A. 2003. Predicting language outcome in infants with autism and pervasive developmental disorder. International Journal of Language and Communication Disorders 38:265-285.

Dohmen, A., Chiat, S., \& Roy, P. (2013). Nonverbal imitation skills in children with specific language delay. Research in Developmental Disabilities, 34(10), 3288-3300.

Enard, W. (2011). FOXP2 and the role of cortico-basal ganglia circuits in speech and language evolution. Current Opinion in Neurobiology, 21(3), 415-424. doi: 10.1016/j.conb.2011.04.008

Fadiga, L., Craighero, L., Destro, M. F., Finos, L., Cotillon-Williams, N., Smith, A. T., \& Castiello, U. (2006). Language in shadow. Social Neuroscience, 1(2), 77-89.

Fawcett, A. J., \& Nicolson, R. I. (1995). Persistent deficits in motor skill for children with dyslexia. Journal of Motor Behavior, 27(3), 235-240.

Fawcett, A. J., \& Nicolson, R. I. (1999). Performance of dyslexic children on cerebellar and cognitive tests. Journal of Motor Behavior, 31(1), 68-78.

Francks, C., Fisher, S. E., Marlow, A. J., MacPhie, I. L., Taylor, K. E., Richardson, A. J., Stein, J. F., \& Monaco, A. P. (2003). Familial and genetic effects on motor coordination, laterality, and reading-related cognition. American Journal of Psychiatry, 160, 1970-1977.

Frederickson, N., Frith, U., \& Reason, R. (1997). Phonological Assessment Battery (PhAB). Windsor, UK: NFER-Nelson.

Gabriel, A., Maillart, C., Stefanik, N., Lejeune, C., Desmotte, L., \& Meulemans, T. (2013). Procedural learning in specific language impairment: Effects of sequence complexity. Journal of the International Neuropsychological Society, 19(3), 264-271. 
507

508

509

510

511

512

513

514

515

516

517

518

519

520

521

522

523

524

525

526

527

528

529

530

531

532

533

534

535

536

537

538

539

540

541

542

543

544

545

546

547

548

549

550

551

552

553

Gaysina, D., Maughan, B., \& Richards, M. (2010). Association of reading problems with speech and motor development: Results from a British 1946 birth cohort. Developmental Medicine \& Child Neurology, 52(7), 680-681.

Heiser, M., Iacoboni, M., Maeda, F., Marcus, J., \& Mazziotta, J. C. (2003). The essential role of Broca's area in imitation. European Journal of Neuroscience, 17(5), 1123-1128.

Hill, E. L. (1998). A dyspraxic deficit in specific language impairment and developmental coordination disorder? Evidence from hand and arm movements. Developmental Medicine \& Child Neurology, 40(6), 388-395.

Hill, E. L. (2001). Non-specific nature of specific language impairment: a review of the literature with regard to concomitant motor impairments. International Journal of Language \& Communication Disorders, 36(2), 149-171.

Hill, E. L., Bishop, D. V. M., \& Nimmo-Smith, I. (1998). Representational gestures in developmental coordination disorder and specific language impairment: Error-types and the reliability of ratings. Human Movement Science, 17(4-5), 655-678.

Hsu, J. H., \& Bishop, D. V. M. (2013). Sequence-specific procedural learning deficits in children with Specific Language Impairment. Developmental Science.

Irannejad, S., \& Savage, R. (2012). Is a cerebellar deficit the underlying cause of reading disabilities? Annals of Dyslexia, 62(1), 22-52.

Kail, R. (1994). A method for studying the generalized slowing hypothesis in children with specific language impairment. Journal of Speech and Hearing Research, 37, 418-421.

Kenny, D. A., Kashy, D. A., \& Cook, W. L. (2006). Dyadic Data Analysis. New York: The Guildford Press.

Korkman, M., Kirk, U., \& Kemp, S. I. (1998). NEPSY: A developmental neuropsychological assessment. San Antonio, TX: Psychological Corporation.

Lum, J. A. G., Conti-Ramsden, G., Page, D., \& Ullman, M. T. (2012). Working, declarative and procedural memory in specific language impairment. Cortex, 48(9), 1138-1154. doi:10.1016/j.cortex.2011.06.001

Lum, J. A. G., Gelgic, C., \& Conti-Ramsden, G. (2010). Procedural and declarative memory in children with and without specific language impairment. International Journal of Language \& Communication Disorders, 45(1), 96-107.

Mayor-Dubois, C., Zesiger, P., Van der Linder, M., \& Roulet-Perez, E. (2012). Nondeclarative learning in children with specific language impairment: Predicting regularities in the visuomotor, phonological, and cognitive domains. Child Neuropsychology, iFirst, 1-9. doi:10.1080/09297049.2012.734293

Monzée, J., Drew, T., \& Smith, A. M. (2004). Effects of muscimol inactivation of the cerebellar nuclei on precision grip. Journal of Neurophysiology, 91(3), 1240-1249.

Morris, R. D., Stuebing, K. K., Fletcher, J. M., Shaywitz, S. E., Lyon, G. R., Shankweiler, D. P., Katz, L., Francis, D. J., \& Shaywitz, B. A. (1998). Subtypes of reading disability: Variability around a phonological core. Journal of Educational Psychology, 90(3), 347-373.

Neale, M. D. (1997). Neale Analysis of Reading Ability (2nd ed.). Windsor, UK: NFER-Nelson.

Needle, J. L., Fawcett, A. J., \& Nicolson, R. I. (2006). Balance and dyslexia: An investigation of adults' abilities. European Journal of Cognitive Psychology, 18(6), 909-936.

Nicolson, R. I., \& Fawcett, A. J. (2007). Procedural learning difficulties: reuniting the developmental disorders? TRENDS in Neurosciences, 30(4), 135-141.

Petrill, S. A., Pike, A., Price, T., \& Plomin, R. (2004). Chaos in the home and socioeconomic status are associated with cognitive development in early childhood: Environmental mediators identified in a genetic design. Intelligence, 32(5), 445-460. 
554

555

556

557

558

559

560

561

562

563

564

565

566

567

568

569

570

571

572

573

574

575

576

577

578

579

580

581

582

583

584

585

586

587

588

589

590

591

592

593

594

595

596

597

598

599

600

Raberger, T., \& Wimmer, H. (2003). On the automaticity/cerebellar deficit hypothesis of dyslexia: balancing and continuous rapid naming in dyslexic and ADHD children. Neuropsychologia, 4l(11), 1493-1497.

Ramus, F. (2004). Neurobiology of dyslexia: A reinterpretation of the data. TRENDS in Neurosciences, 27(12), 720-726.

Ramus, F., Pidgeon, E., \& Frith, U. (2003). The relationship between motor control and phonology in dyslexic children. Journal of Child Psychology and Psychiatry, 44(5), 712722.

Rochelle, K. S. H., \& Talcott, J. B. (2006). Impaired balance in developmental dyslexia? A metaanalysis of the contending evidence. Journal of Child Psychology and Psychiatry, 47(11), $1159-1166$.

Rutter, M., \& Yule, W. (1970). Neurological aspects of intellectual retardation and specific reading retardation. In M. Rutter, J. Tizard, \& K. Whitmore (Eds.), Education, health and behavior (pp. 54-74). London: Longman.

Rutter, M., Caspi, A., Fergusson, D. M., Horwood, L. J., Goodman, R., Maughan, B., Moffitt, T.E, \& Carroll, J. (2004). Gender differences in reading difficulties: findings from four epidemiological studies. Journal of the American Medical Association, 291, 2007-2012.

Savage, R. S., \& Frederickson, N. (2006). Beyond phonology: What else is needed to describe the problems of below-average readers and spellers? Journal of Learning Disabilities, 39(5), 399-413.

Smits-Engelsman, B., \& Hill, E. L. (2012). The relationship between motor coordination and intelligence across the IQ range. Pediatrics, 130(4), e950-e956. doi: 10.1542/peds.20113712

Stoodley, C. J., Harrison, E. P. D., \& Stein, J. F. (2006). Implicit motor learning deficits in dyslexic adults. Neuropsychologia, 44(5), 795-798.

Stoodley, C. J., \& Stein, J. F. (2006). A processing speed deficit in dyslexic adults? Evidence from a peg-moving task. Neuroscience Letters, 399(3), 264-267.

Szmalec, A., Loncke, M., Page, M. P. A., \& Duyck, W. (2011). Order or disorder? Impaired Hebb learning in dyslexia. Journal of Experimental Psychology: Learning, Memory, and Cognition, 37(5), 1270-1279.

Tiffin, J. (1968). Purdue Pegboard examiner's manual. Rosemont, IL: London House.

Tomblin, J. B., Mainela-Arnold, E., \& Zhang, X. (2007). Procedural learning in adolescents with and without specific language impairment. Language Learning and Development, 3(4), 269-293.

Torgesen, J. K., Wagner, R., \& Rashotte, C. (1999). Test of Word Reading Efficiency (TOWRE). New York: Psychological Corporation.

Trouton, A., Spinath, F. M., \& Plomin, R. (1994). Twins Early Development Study (TEDS): A multivariate, longitudinal genetic investigation of language, cognition and behavior problems in childhood. Twin Research, 5(5), 444-448.

Trzesniewski, K. H., Moffitt, T. E., Caspi, A., Taylor, A., \& Maughan, B. (2006). Revisiting the association between reading achievement and antisocial behavior: New evidence of an environmental explanation from a twin study. Child Development, 77(1), 72-88.

Ullman, M. T., \& Pierpont, E. I. (2005). Specific language impairment is not specific to language: The procedural deficit hypothesis. Cortex, 41(3), 399-433.

Vukovic, M., Vukovic, I., \& Stojanovik, V. (2010). Investigation of language and motor skills in Serbian speaking children with specific language impairment and in typically developing children. Research in Developmental Disabilities, 31, 1633-1644. 
601 Wechsler, D. (1999). Wechsler Abbreviated Scale of Intelligence. San Antonio, TX: Psychological 602 Corporation.

603 White, S., Milne, E., Rosen, S., Hansen, P., Swettenham, J., Frith, U., \& Ramus, F. (2006). The 604 role of sensorimotor impairments in dyslexia: A multiple case study of dyslexic children.

605 Developmental Science, 9(3), 237-255.

606 Williams, J. H. G., Whiten, A., Suddendorf, T., \& Perrett, D. I. (2001). Imitation, mirror neurons 607 and autism. Neuroscience and Biobehavioral Reviews, 25(4), 287-295.

608 Wimmer, H., Mayringer, H., \& Landerl, K. (1998). Poor reading: A deficit in skill-automatization 609 or a phonological deficit. Scientific Studies of Reading, 2(4), 321-340.

610 Woodcock, R. W., McGrew, K. S., \& Mather, N. (2001). Woodcock Johnson III. Itasca, IL:

611 Riverside Publishing. 


\section{Table 1 (on next page)}

Means (SDs) on selection and background variables for four groups

TD: Typically developing; $\mathrm{RD}=$ reading disabled; $\mathrm{LI}=$ language impaired. Means with different superscripts differ significantly at the .05 level on LSD test after adjustment of degrees of freedom for twin as random factor. 


\begin{tabular}{|c|c|c|c|c|c|c|}
\hline & Group & TD & $\mathrm{RD}$ & LI & $\mathrm{LI}+\mathrm{RD}$ & Anova output \\
\hline \multirow[t]{2}{*}{ Test } & $\mathrm{N}$ & 117 & 67 & 32 & 37 & \\
\hline & $\%$ male & 40 & 49 & 56 & 68 & \\
\hline \multicolumn{7}{|l|}{ Nonverbal Ability } \\
\hline \multirow[t]{2}{*}{ WASI Block Design } & Mean & 97.8 & 99.1 & 95.3 & 96.9 & $\mathrm{~F}(3,246.6)=0.9$ \\
\hline & $\mathrm{SD}$ & 11.87 & 11.93 & 10.99 & 11.57 & $\mathrm{p}=.439$ \\
\hline \multicolumn{7}{|l|}{ Language } \\
\hline \multirow[t]{2}{*}{ WASI Vocabulary } & Mean & $98.2^{\mathrm{a}}$ & $93.3^{b}$ & $83.0^{\mathrm{c}}$ & $78.9^{c}$ & $F(3,223.7)=33.37$ \\
\hline & SD & 13.17 & 13.05 & 11.53 & 12.39 & $\mathrm{p}<.001$ \\
\hline WJ Understanding & Mean & $99.6^{\mathrm{a}}$ & $95.9^{\mathrm{a}}$ & $78.2^{\mathrm{b}}$ & $83.9^{\mathrm{b}}$ & $F(3,246.9)=31.56$ \\
\hline \multicolumn{7}{|l|}{ Directions } \\
\hline & SD & 13.57 & 13.62 & 12.88 & 13.35 & $\mathrm{p}<.001$ \\
\hline \multirow[t]{2}{*}{ ERRNI Comprehension } & Mean & $98.6^{\mathrm{a}}$ & $98.8^{\mathrm{a}}$ & $91.8^{\mathrm{b}}$ & $88.0^{\mathrm{b}}$ & $F(3,242)=6.87$ \\
\hline & SD & 14.55 & 14.65 & 14.13 & 14.48 & $\mathrm{p}<.001$ \\
\hline \multirow[t]{2}{*}{ ERRNI MLU } & Mean & $102.1^{\mathrm{a}}$ & $97.6^{\mathrm{a}}$ & $89.5^{\mathrm{b}}$ & $87.8^{\mathrm{b}}$ & $\mathrm{F}(3,224.1)=11.53$ \\
\hline & SD & 15.47 & 15.72 & 15.2 & 15.22 & $\mathrm{p}<.001$ \\
\hline NEPSY Sentence & Mean & $97.1^{\mathrm{a}}$ & $92.0^{\mathrm{b}}$ & $81.1^{c}$ & $74.7^{\mathrm{d}}$ & $F(3,243.6)=38.66$ \\
\hline \multicolumn{7}{|l|}{ Repetition } \\
\hline \multicolumn{7}{|l|}{ Reading } \\
\hline \multirow[t]{2}{*}{ TOWRE word reading } & Mean & $102.6^{\mathrm{a}}$ & $71.4^{\mathrm{c}}$ & $97.6^{\mathrm{b}}$ & $68.9^{c}$ & $F(3,242.8)=150.84$ \\
\hline & SD & 11.54 & 11.61 & 11.2 & 11.48 & $\mathrm{p}<.001$ \\
\hline \multirow{2}{*}{$\begin{array}{l}\text { TOWRE phonemic } \\
\text { decoding }\end{array}$} & Mean & $101.2^{\mathrm{a}}$ & $75.0^{\mathrm{c}}$ & $95.3^{\mathrm{b}}$ & $73.1^{\mathrm{c}}$ & $F(3,247.1)=114.37$ \\
\hline & SD & 11.06 & 11.15 & 10.53 & 11.1 & $\mathrm{p}<.001$ \\
\hline \multicolumn{7}{|l|}{ Family Background } \\
\hline \multirow[t]{2}{*}{ SES index } & Mean & -0.01 & -0.06 & -0.24 & -0.21 & $F(3,252)=1.36$ \\
\hline & SD & 0.710 & 0.710 & 0.584 & 0.693 & $\mathrm{p}=.255$ \\
\hline
\end{tabular}




\section{Table 2 (on next page)}

Statistics for main effects and interaction of LI/RD status on four motor tasks

Note: * denotes $p<.05$ 


\begin{tabular}{|c|c|c|c|c|c|}
\hline Effect & Statistic & $\begin{array}{l}\text { Finger } \\
\text { tapping }\end{array}$ & $\begin{array}{l}\text { Finger } \\
\text { sequences }\end{array}$ & $\begin{array}{l}\text { Purdue } \\
\text { pegboard }\end{array}$ & $\begin{array}{l}\text { Imitation of } \\
\text { hand positions }\end{array}$ \\
\hline \multirow[t]{4}{*}{ LI } & $\mathrm{F}$ & 0.07 & 0.06 & $5.85^{*}$ & $6.42 *$ \\
\hline & $\mathrm{DF}$ & $1,246.6$ & $1,245.9$ & $1,247.8$ & $1,238.8$ \\
\hline & $\mathrm{p}$ & .796 & .812 & .016 & .012 \\
\hline & Cohen's d & .034 & .030 & .316 & .318 \\
\hline \multirow[t]{4}{*}{$\mathrm{RD}$} & $\mathrm{F}$ & 0.02 & 3.0 & 0.92 & 0.48 \\
\hline & $\mathrm{DF}$ & $1,247.4$ & $1,247.8$ & $1,245.8$ & $1,247.0$ \\
\hline & $\mathrm{p}$ & .900 & .084 & .338 & .488 \\
\hline & Cohen's d & .017 & .208 & .116 & .082 \\
\hline \multirow[t]{3}{*}{$\mathrm{LI} \times \mathrm{RD}$} & $\mathrm{F}$ & 1.91 & 0.05 & 0.11 & 0.03 \\
\hline & DF & $1,226.2$ & $1,224.0$ & $1,230.9$ & $1,209.4$ \\
\hline & $\mathrm{p}$ & .169 & .830 & .736 & .874 \\
\hline \multirow[t]{3}{*}{ Sex } & $\mathrm{F}$ & 2.78 & 0.57 & 0.56 & 2.49 \\
\hline & $\mathrm{DF}$ & $1,152.1$ & $1,153.04$ & $1,148.5$ & $1,151.8$ \\
\hline & $\mathrm{p}$ & .098 & .452 & .454 & .116 \\
\hline
\end{tabular}




\section{Figure 1}

Mean scores on four motor tasks

Error bars show standard errors 

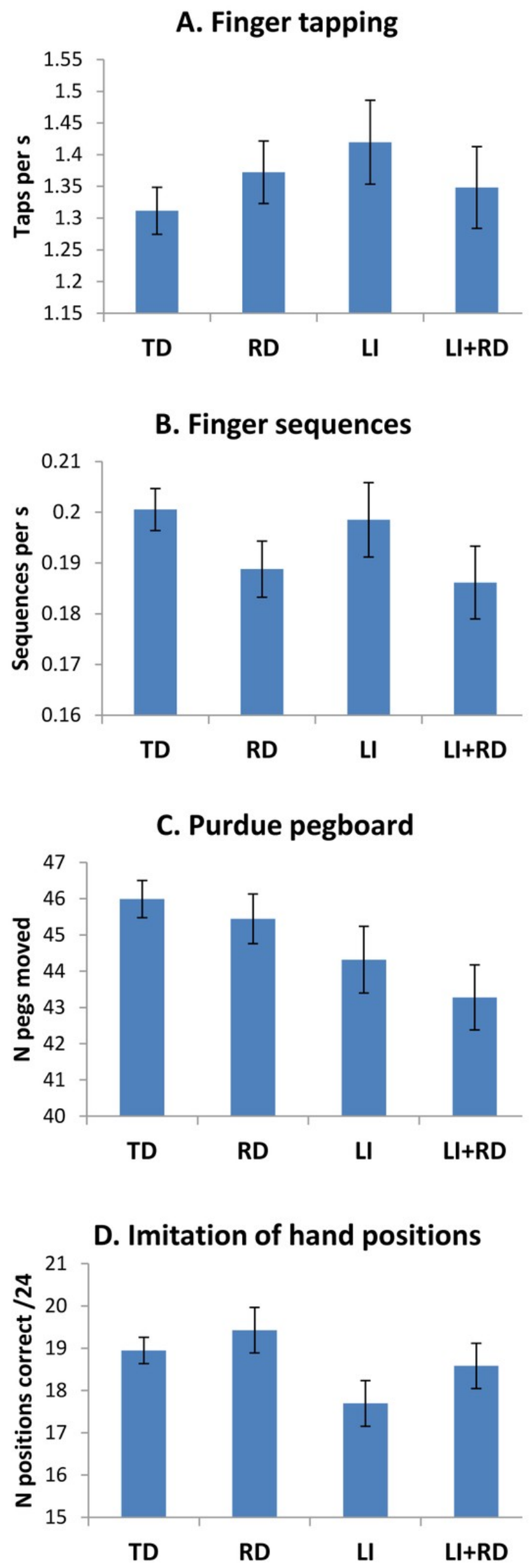

PeerJ reviewing PDF | (v2013:07:671:3:0:NEW 6 Nov 2013) 


\section{Figure 2}

Correlations of four motor tasks with measures of language and literacy

Block design (nonverbal ability) has been partialled out. Correlations extending beyond the bold line are significant at $p<.05$. Those extending beyond the dotted line are significant at $p$ $<.01$.

\section{A. Finger Tapping}

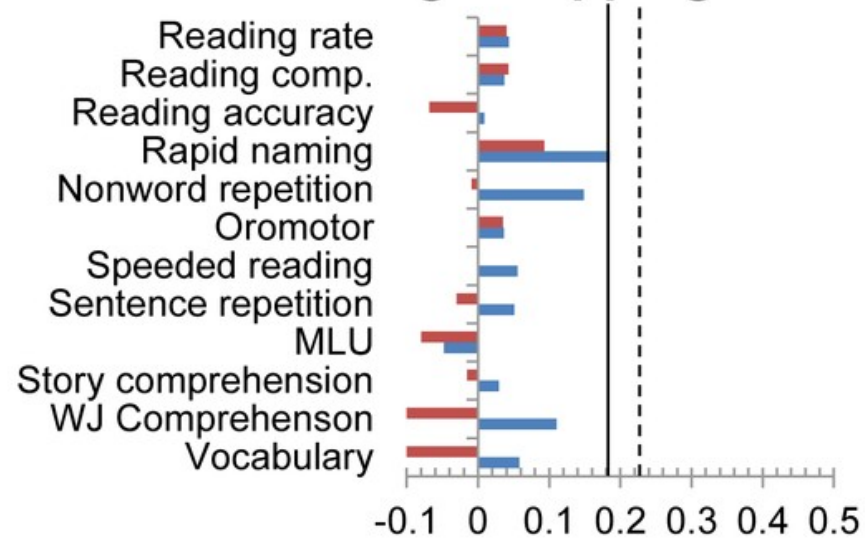

\section{Purdue Pegboard}

Reading rate Reading comp. Reading accuracy

Rapid naming

Nonword repetition Oromotor

Speeded reading Sentence repetition MLU

Story comprehension WJ Comprehenson Vocabulary

\section{B. Finger Sequences}

Reading rate Reading comp. Reading accuracy Rapid naming Nonword repetition Oromotor Speeded reading Sentence repetition MLU Story comprehension WJ Comprehenson Vocabulary

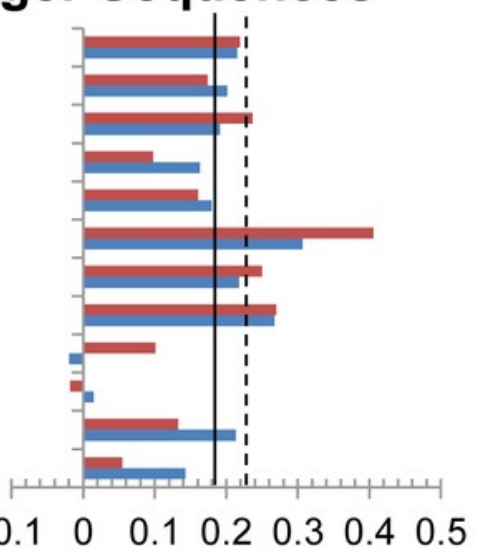

\section{Imitating hand positions}

Reading rate Reading comp. Reading accuracy Rapid naming Nonword repetition Oromotor Speeded reading Sentence repetition

Story comprehension WJ Comprehenson Vocabulary

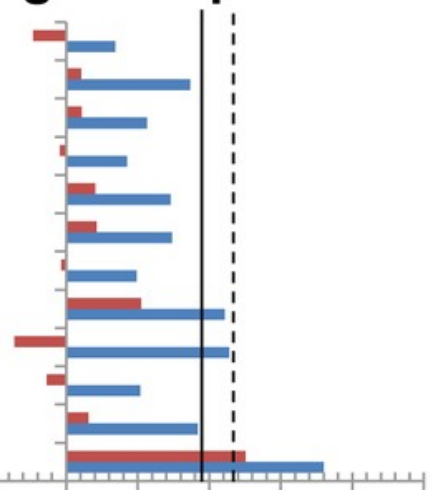

$\begin{array}{lllllll}-0.1 & 0 & 0.1 & 0.2 & 0.3 & 0.4 & 0.5\end{array}$ Pearson correlation (r) twin 1

twin 2 